\title{
10 Choice of journal
}

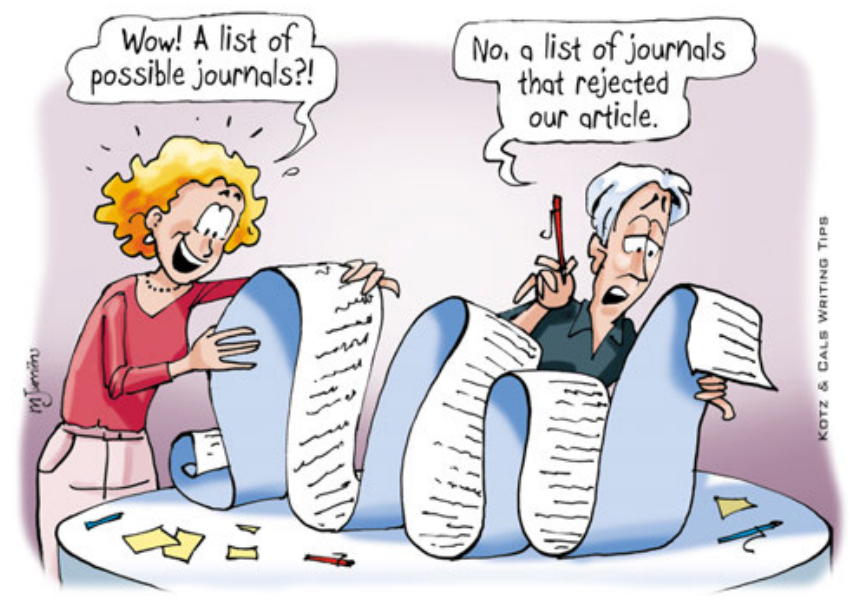

Tip 10 - Choice of journal: define a list of target journals!

\section{What you should know}

In a scientific paper you try to tell a story, but to whom? Your audience will largely depend on the journal you publish your work in. PubMed alone cites over 30 million papers from 7,000 scientific journals. This shows that with some perseverance you will probably get your paper published at some point, but choosing the right journal for your work is not easy.

Depending on a journal's status, space restrictions and flow of submitted papers, acceptance rates vary from under $10 \%$ for the most prestigious journals to over $80 \%$ for some journals. The impact factor of a journal reflects the average number of citations of articles published in the journal in the past two years, and is a much debated but still widely used measure of a journal's relative importance in the field, published in the annual Journal Citation Reports. These also allow you to browse journals by subject category, which is useful to get an overview of journals within a specific field.

While traditional scientific journals will often publish printed issues alongside electronic versions on their website, the number of electronic journals without printed formats is ever increasing. Such journals are not constricted by 
space limitations, and will often be able to publish more papers a year as well as being able to publish papers very soon after acceptance.

Many scientific journals are nowadays (partly) open-access. This is the practice of providing unrestricted access to and unrestricted reuse of peer-reviewed scholarly research via the Internet, so papers are also accessible to people who do not subscribe to the journal (e.g., researchers not attached to an academic department or living in low-income countries), and materials may be used and copied, subject to proper attribution of authorship. Open access does not necessarily mean that the author must pay a publication fee upon acceptance, although many journals (traditional as well as electronic) do charge such fees (often around $€ 2,000$ ), which are replacing the publishers' traditional business model of reader subscription fees. Be aware that electronic publishing does not automatically imply open-access publishing, or vice versa.

Unfortunately, there is an increasing numer of predatory publishers who send out e-mail invitations to submit papers, while only being interested in pocketing publication fees. These journals charge publication fees to authors without checking articles for quality and legitimacy. Proper scientific journals will not normally send such e-mails. Google "predatory journals" for an update on which journals and publishers can't be trusted.

\section{What you should do}

Think about a target journal before starting to write. The nature of the target journal will affect the outline of your paper in general and the introduction and discussion sections in particular (see part 1: how to get started). Discuss within your team the focus and preferred audience for this specific paper. Look at the relevant literature for your project to see where similar work has been published. Decide if you want to reach fellow researchers, health professionals or both, and draw up a list of general journals or more disease-specific or contentspecific specialist journals. Be realistic about the likeliness of your paper being accepted by a top journal. Ask your (senior) peers for an honest opinion and also ask them about their experiences with journals' peer-review and publication process in terms of punctuality and reasons for rejection or acceptance. Look at the journals' websites and author's instruction to see if a journal actually publishes the type of paper you intend to write (e.g., some may or may not publish reviews, qualitative research or short reports).

Balance the desire to publish in high impact factor journals with your preferred time to publication. Submitting a paper to a journal with a high rejection rate may give you useful reviewer reports (see part 12: responding to reviewers), 
but may also delay your publication process by several months as multiple submissions may be necessary. Once you hit the submit button, the paper will be off your desk for quite a while. Posting your paper on a pre-print server can be considered, especially when doing research in a quickliy evolving field. By posting pre-prints, authors can help promote openness and transparency and reduce research waste from duplicated efforts and non-reporting. The best known pre-print server in biomedical research is MedRxiv. If you want to publish in a specific journal, be sure to read the journal. Look carefully at the table of contents and the papers, and read editorials to learn about the scope of the journal and the editors' preferences. This will give you a better estimate of your chances of getting published and may also enable you to adapt your manuscript.

Draw up a list of relevant target journals and discuss it with the author team. Then prioritise and rank a final list of 3-5 journals. This will enable you to focus on your first journal of choice and adhere to their author's instructions, while also giving you a predefined strategy should the paper be rejected by the first journal.

\section{Checklist for choice of journal}

- Think about a target journal before you start writing

- Consider the following journal characteristics:

- scope (fitting the papers' primary focus)

- basic vs. clinical research

- generalist vs. specialist journal

- traditional (printed) vs. electronic journal

- $\quad$ subscription access vs. open access

- Balance the desire to publish in top-quality journals with the need for rapid publication

- Consider, but don't be fooled, by impact factors

- Be aware of predatory publishers: don't be fooled by e-mail invitations.

- Read the journals you aim to publish in to learn about their preferences

- Draw up a prioritised list of 3-5 journals

- Consider publishing your paper on a pre-print server as well 